Pacific Journal of Mathematics

ON THE FIX-POINTS OF COMPOSITE FUNCTIONS 


\title{
ON THE FIX-POINTS OF COMPOSITE FUNCTIONS
}

\section{WALTER BERGWEILER}

\begin{abstract}
Gross has conjectured that a composite transcendental entire function has infinitely many fix-points. We show that the conjecture is true if one of the two components has finite order.
\end{abstract}

1. Introduction and results. Let $f$ and $g$ be two nonlinear entire functions, at least one of them transcendental. Gross [4] has conjectured that the composite function $f \circ g$ has infinitely many fix-points.

Gross and Osgood [5] have proved that the conjecture is true, if one of the functions $f$ and $g$ is of finite order while the other one is of finite lower order. The conjecture has also been proved under various other conditions on $f$ and $g$ (cf. [6], [9], [13], [14]).

We shall prove

THEOREM 1. Let $f$ and $g$ be nonlinear entire functions, at least one of them transcendental. If one of the functions $f$ and $g$ is of finite order, then $f \circ g$ has infinitely many fix-points.

As a consequence of Theorem 1 we obtain

THEOREM 2. Let $f$ and $g$ be nonlinear entire functions, at least one of them transcendental. If

$$
\limsup _{r \rightarrow \infty} \frac{\log \log \log M(r, f \circ g)}{\log r}<\infty,
$$

then $f \circ g$ has infinitely many fix-points.

These two theorems contain and generalize many of the results referred to above.

2. Lemmas. Our proofs will be based partially on Nevanlinna theory (for notations see [7]), but mainly on Wiman-Valiron theory. We denote the maximum term of an entire function $h$ by $\mu(r, h)$ and the central index by $N=N(r, h)$. By $F$ we denote an exceptional set of finite logarithmic measure, not necessarily the same at each occurrence. For the convenience of the reader we state the results of 
Wiman-Valiron theory that we need. In fact Hayman [8] has obtained much more precise estimations, but the following results suffice for our purposes.

LEMMA $1([\mathbf{8}]$, see also [12]). Let $h$ be entire, $k>0, \gamma>1 / 2$, $0<\eta<1$ and $\varepsilon>0$. Assume that $\left|z_{0}\right|=r,\left|h\left(z_{0}\right)\right| \geq \eta M(r, h)$ and $|\tau| \leq k N^{-\gamma}$. Then

$$
\begin{gathered}
h\left(z_{0} e^{\tau}\right) \sim h\left(z_{0}\right) e^{N \tau} \quad(r \notin F), \\
h^{\prime}\left(z_{0} e^{\tau}\right) \sim \frac{N}{z_{0} e^{\tau}} h\left(z_{0}\right) e^{N \tau} \quad(r \notin F), \\
\log \mu(r, h) \sim \log M(r, h) \sim \log M\left(r, h^{\prime}\right) \quad(r \notin F), \\
N \leq(\log \mu(r, h))^{1+\varepsilon} \quad(r \notin F), \\
\log \mu(r, h) \leq N \log r+O(1) .
\end{gathered}
$$

LEMMA 2. Let $h$ be entire, $K>0,0<\eta<1$ and $\varepsilon>0$. If $\left|\sigma_{1}\right|<K,\left|h\left(z_{0}\right)\right| \geq \eta M(r, h)$ and if $\left|z_{0}\right|=r \notin F$ is large enough, then there exists $\tau_{1}$ such that $\left|N \tau_{1}-\sigma_{1}\right|<\varepsilon$ and $h\left(z_{0} e^{\tau_{1}}\right)=h\left(z_{0}\right) e^{\sigma_{1}}$. If $\varepsilon<2 \pi$ and if $r \notin F$ is large enough, then $\tau_{1}$ is unique.

Proof. Put $w_{1}=h\left(z_{0}\right) e^{\sigma_{1}}$ and consider $f_{1}(\tau)=h\left(z_{0} e^{\tau}\right)$ and $f_{2}(\tau)$ $=h\left(z_{0}\right) e^{N \tau}=w_{1} \exp \left(N \tau-\sigma_{1}\right)$. If $\left|N \tau-\sigma_{1}\right|=\varepsilon$, then

$$
f_{1}(\tau) \sim h\left(z_{0}\right) e^{N \tau}=f_{2}(\tau)
$$

by (2.1) and therefore

$$
\left|\left(f_{1}(\tau)-w_{1}\right)-\left(f_{2}(\tau)-w_{1}\right)\right|=\left|f_{1}(\tau)-f_{2}(\tau)\right|=o\left(\left|f_{2}(\tau)\right|\right) .
$$

On the other hand, we have for $\left|N \tau-\sigma_{1}\right|=\varepsilon$

$$
\begin{aligned}
\left|f_{2}(\tau)-w_{1}\right| & =\left|w_{1}\left(\exp \left(N \tau-\sigma_{1}\right)-1\right)\right| \\
& \geq \delta_{1}\left|w_{1}\right| \geq \delta_{2}\left|f_{2}(\tau)\right|
\end{aligned}
$$

for some $\delta_{1} \geq \delta_{2}>0$, if $0<\varepsilon<2 \pi$. The conclusion follows from (2.6) and (2.7) by Rouché's theorem.

Clunie [3] has given the following application.

LEMMA 3. If $f$ and $g$ are entire, then

$$
M(r, f \circ g)=M((1+o(1)) M(r, g), f) \quad(r \notin F) .
$$


Next we note that if $f \circ g$ has only a finite number of fix-points, then

$$
f(g(z))=P(z) e^{\alpha(z)}+z
$$

where $\alpha$ is an entire function and $P$ is a polynomial. A consequence of Lemma 3 is

LEMMA 4. If (2.9) holds, then

$$
M(r, \alpha) \sim \log M((1+o(1)) M(r, g), f) \quad(r \notin F) .
$$

The following lemma is implicit in the work of Gross and Osgood [5].

LEMMA 5. If (2.9) holds, then

$$
T(r, g)=o\left(T\left(r, \alpha^{\prime}\right)\right) \quad(r \notin E),
$$

where $E$ has finite linear measure.

In fact, if $T\left(r, \alpha^{\prime}\right) \leq K T(r, g)$ for a constant $K$ on a set of infinite measure, then a modification of a theorem of Steinmetz [11] (cf. [5]) yields that $f$ satisfies a certain differential equation. As shown in [5], this leads to a contradiction.

We remark that for our purposes the weaker inequality

$$
T(r, g)=O\left(T\left(r, \alpha^{\prime}\right)\right) \quad(r \notin E)
$$

will be sufficient. This inequality is easier to obtain than (2.11), in fact the method used in [2] for the Riccati equation applies also to the linear equation

$$
\begin{aligned}
\frac{d}{d z}(f(g(z)))= & \left(\frac{P^{\prime}(z)}{P(z)}+\alpha^{\prime}(z)\right) f(g(z)) \\
& -\left(\frac{P^{\prime}(z)}{P(z)}+\alpha^{\prime}(z)\right) z+1,
\end{aligned}
$$

which is a consequence of (2.9).

We also need

LEMMA 6 [1]. Let $h(x)$ and $k(x)$ be non-negative, non-decreasing and convex for $x \geq 0$. Let $K>1$ and suppose that $h(x) \leq k(x)$ for all $x \geq 0$. Then $h^{\prime}(x) \leq K k^{\prime}(x)$ on a set of lower density at least $(K-1) / K$. 
A consequence is

LEMMA 7. Let $\alpha$ and $g$ be entire functions, $c>0, K>1$ and assume that

$$
\log M(r, \alpha)<c \log M(r, g) \quad(r \notin F) .
$$

Then

$$
N(r, \alpha) \leq K c N(r, g)
$$

on a set of positive lower logarithmic density.

Proof. Let $\varepsilon>0$ and put $x=\log r, h(x)=\max \{0, \log \mu(r, \alpha)\}$, $k(x)=\max \{h(x),(c+\varepsilon) \log \mu(r, g)\}$. The conclusion follows from (2.3) and Lemma 6, since

$$
\frac{d \mu(r, h)}{d \log r}=N(r, h)
$$

for an entire function $h$, except for the discontinuities of $N(r, h)$.

\section{Proof of Theorems.}

Proof of Theorem 1 . Since $f \circ g$ has infinitely many fix-points if and only if $g \circ f$ does [6, p. 214, proof of Theorem 2], we may assume that the order of $f$ is finite. The conclusion follows from the result of Gross and Osgood [5] mentioned in the introduction, if the lower order of $g$ is finite. Hence we may assume that the lower order of $g$ is infinite. What we need, however, is only that $g$ has non-zero lower order.

Suppose that $f \circ g$ has only a finite number of fix-points, so that (2.9) holds. Lemma 4 shows that $\log M(r, \alpha)=O(\log M(r, g))$ for $r \notin F$ and Lemma 7 implies that there exists a positive constant $c$ such that

$$
N(r, \alpha) \leq c N(r, g) \quad(r \in H)
$$

where $H$ has positive lower logarithmic density. It follows easily from a classical lemma due to Borel [7, Lemma 2.4] that for $\beta>0$

$$
\log M(r, g) \leq T(r, g)^{1+\beta} \quad(r \notin E),
$$

where $E$ has finite linear measure. Combining (2.3), (2.4), (2.5), (2.12) and (3.2), we get for $\varepsilon>0$ and $r \notin F$

$$
\begin{aligned}
N(r, g) & \leq[\log \mu(r, g)]^{1+\varepsilon} \leq[\log M(r, g)]^{1+\varepsilon} \\
& \leq T(r, g)^{1+2 \varepsilon} \leq T\left(r, \alpha^{\prime}\right)^{1+3 \varepsilon} \leq\left[\log M\left(r, \alpha^{\prime}\right)\right]^{1+3 \varepsilon} \\
& \leq[\log \mu(r, \alpha)]^{1+4 \varepsilon} \leq[N(r, \alpha) \log r]^{1+5 \varepsilon}
\end{aligned}
$$


From the assumption that the lower order of $g$ is positive (or infinite) we can deduce that

$$
\log r \leq N(r, g)^{\varepsilon},
$$

if $r$ is large enough. If $1 / 2<\gamma<1$ and if $\varepsilon>0$ is suitably chosen, then (3.3) and (3.4) imply that

$$
N(r, g)^{\gamma} \leq N(r, \alpha) \quad(r \notin F) .
$$

Now choose $z_{0}$ such that $\left|f\left(g\left(z_{0}\right)\right)\right|=M(r, f \circ g)$, where $r=\left|z_{0}\right|$. It follows from Lemma 3 that

$$
\left|g\left(z_{0}\right)\right|=(1-o(1)) M(r, g) \quad(r \notin F)
$$

and that

$$
M\left(r, e^{\alpha}\right)=\exp ((1-o(1)) M(r, \alpha)) \quad(r \notin F) .
$$

If we put $m(r, P)=\min \{|P(z)| ;|z|=r\}$, where $P$ is the polynomial from the representation $(2.9)$, then

$$
\begin{aligned}
M\left(r, e^{\alpha}\right) & =M\left(r, \frac{P e^{\alpha}+z-z}{P}\right) \leq \frac{M\left(r, P e^{\alpha}+z\right)+r}{m(r, P)} \\
& =\frac{\left|P\left(z_{0}\right) e^{\alpha\left(z_{0}\right)}+z_{0}\right|+r}{m(r, P)} \leq \frac{M(r, P)}{m(r, P)}\left|e^{\alpha\left(z_{0}\right)}\right|+\frac{2 r}{m(r, P)} \\
& =(1+o(1)) \exp \left(\operatorname{Re} \alpha\left(z_{0}\right)\right) .
\end{aligned}
$$

Combining (3.7) and (3.8) we get

$$
\left|\alpha\left(z_{0}\right)\right| \geq \operatorname{Re} \alpha\left(z_{0}\right) \geq(1-o(1)) M(r, \alpha) \quad(r \notin F) .
$$

Lemma 2 implies that there exists $\tau_{1}$ satisfying $\left|\tau_{1} N(r, g)-2 \pi i\right|=$ $o(1)$ such that $g\left(z_{0} e^{\tau_{1}}\right)=g\left(z_{0}\right)$, provided $r \notin F$. Let $z_{1}=z_{0} e^{\tau_{1}}$ and

$$
l(z)=\frac{f^{\prime}(g(z)) g^{\prime}(z)-1}{f(g(z))-z} .
$$

Then

$$
\frac{l\left(z_{1}\right)}{l\left(z_{0}\right)} \sim \frac{g^{\prime}\left(z_{1}\right)}{g^{\prime}\left(z_{0}\right)} \sim \frac{g\left(z_{1}\right)}{g\left(z_{0}\right)}=1
$$

by (3.6) and Lemma 1. On the other hand we have

$$
l(z)=\frac{P^{\prime}(z)}{P(z)}+\alpha^{\prime}(z)
$$


by (2.9). Since

$$
\left|\tau_{1}\right| \leq \frac{2 \pi+o(1)}{N(r, g)} \leq \frac{2 \pi c+o(1)}{N(r, \alpha)} \quad(r \in H \backslash F)
$$

by (3.1), we have

$$
\frac{l\left(z_{1}\right)}{l\left(z_{0}\right)} \sim \frac{\alpha^{\prime}\left(z_{1}\right)}{\alpha^{\prime}\left(z_{0}\right)} \sim \frac{\alpha\left(z_{1}\right)}{\alpha\left(z_{0}\right)} \sim \exp \left(\tau_{1} N(r, \alpha)\right) \quad(r \in H \backslash F)
$$

by (3.9) and Lemma 1. It follows from (3.10) and (3.11) that $\tau_{1} N(r, \alpha)=2 \pi i k+o(1)$ for some integer $k=k(r)$, provided $r \in$ $H \backslash F$. Hence we have

$$
\frac{N(r, \alpha)}{N(r, g)} \sim k(r) \in \mathbf{Z} \quad(r \in H \backslash F)
$$

where $k(r) \leq c$ by $(3.1)$.

Now let $\tau_{2}=i \pi / N(r, \alpha)$ and $z_{2}=z_{0} e^{\tau_{2}}$. Lemma 1 and (3.9) imply that $\alpha\left(z_{2}\right) \sim\left(-\alpha\left(z_{0}\right)\right)$ and $\operatorname{Re} \alpha\left(z_{2}\right) \sim(-M(r, \alpha))$ for $r \notin F$. It follows from (3.5) that $\left|\tau_{2}\right| \leq \pi N(r, g)^{-\gamma}$ for $r \notin F$. Hence we have

$$
\left|g\left(z_{2}\right)\right| \sim\left|g\left(z_{0}\right) \exp \left(N(r, g) \tau_{2}\right)\right| \sim\left|g\left(z_{0}\right)\right| \sim M(r, g) \quad(r \notin F)
$$

by Lemma 1 . Lemma 2 implies that there exists $\tau_{3}$ satisfying $\left|\tau_{3} N(r, g)-2 \pi i\right|=o(1)$ such that $g\left(z_{2} e^{\tau_{3}}\right)=g\left(z_{2}\right)$. Let $z_{3}=z_{2} e^{\tau_{3}}$. To estimate $\alpha\left(z_{3}\right)$ we note that

$$
\left|\tau_{3}\right| \leq \frac{2 \pi c+o(1)}{N(r, \alpha)} \quad(r \in H \backslash F)
$$

by (3.1). Hence Lemma 1 and (3.12) imply that

$$
\begin{aligned}
\alpha\left(z_{3}\right) & \sim \alpha\left(z_{2}\right) \exp \left(N(r, \alpha) \tau_{3}\right) \\
& \sim \alpha\left(z_{2}\right) \exp ((k(r)+o(1))(2 \pi i+o(1))) \\
& \sim \alpha\left(z_{2}\right) \quad(r \in H \backslash F) .
\end{aligned}
$$

Since $g\left(z_{2}\right)=g\left(z_{3}\right)$ we have

$$
z_{2}+P\left(z_{2}\right) e^{\alpha\left(z_{2}\right)}=f\left(g\left(z_{2}\right)\right)=f\left(g\left(z_{3}\right)\right)=z_{3}+P\left(z_{3}\right) e^{\alpha\left(z_{3}\right)} .
$$

It follows that

$$
\begin{aligned}
\left|z_{3}-z_{2}\right| & \leq\left|P\left(z_{2}\right) e^{\alpha\left(z_{2}\right)}\right|+\left|P\left(z_{3}\right) e^{\alpha\left(z_{3}\right)}\right| \\
& \leq r^{K} \exp (-(1-o(1)) M(r, \alpha))
\end{aligned}
$$

for some constant $K$ and $r \in H \backslash F$. On the other hand we have

$$
\left|z_{3}-z_{2}\right|=\left|z_{2}\left(e^{\tau_{3}}-1\right)\right| \sim r\left|\tau_{3}\right| \sim \frac{2 \pi r}{N(r, g)},
$$


so that

$$
N(r, g) \geq(1-o(1)) 2 \pi r^{1-K} \exp ((1-o(1)) M(r, \alpha)) \geq \exp \frac{M(r, \alpha)}{2}
$$

for sufficiently large $r \in H \backslash F$. By (3.3) we have

$$
N(r, g) \leq[\log \mu(r, \alpha)]^{1+4 \varepsilon} \leq[\log M(r, \alpha)]^{1+4 \varepsilon} \quad(r \notin F) .
$$

Altogether we find for $\varepsilon=1 / 4$ that

$$
\exp \frac{M(r, \alpha)}{2} \leq[\log M(r, \alpha)]^{2} \quad(r \in H \backslash F) .
$$

This is an obvious contradiction and the theorem is proved.

Proof of Theorem 2. Assume that $f \circ g$ has only a finite number of fix-points so that (2.9) holds. It is easy to show that

$$
\rho(\alpha)=\limsup _{r \rightarrow \infty} \frac{\log \log \log M\left(r, e^{\alpha}\right)}{\log r},
$$

where $\rho(\alpha)$ denotes the order of $\alpha$. In fact this is a special case of a theorem of Schönhage [10, Satz 6]. It follows from (2.9) and the hypothesis that $\rho(\alpha)<\infty$. Moreover, we have $\rho\left(\alpha^{\prime}\right)=\rho(\alpha)$ and (2.11) or (2.12) imply that $\rho(g) \leq \rho\left(\alpha^{\prime}\right)$. Hence we have $\rho(g)<\infty$, and the conclusion follows from Theorem 1.

Acknowledgment. I am thankful to Professor W. H. J. Fuchs for some valuable discussion on the subject.

\section{REFERENCES}

[1] W. Bergweiler, An inequality for real functions with applications to function theory, Bull. London Math. Soc., 21 (1989), 171-175.

[2] W. Bergweiler, G. Jank and L. Volkmann, Über faktorisierbare Lösungen Riccatischer Differentialgleichungen, Resultate Math., 10 (1986), 40-53.

[3] J. Clunie, The Composition of Entire and Meromorphic Functions, Math. Essays Dedicated to A. J. Macintyre (1970), 75-92, Ohio Univ. Press.

[4] F. Gross, Factorization of Meromorphic Functions, U.S. Government Printing Office, Washington, D.C., 1972.

[5] F. Gross and C. F. Osgood, On fixed points of composite entire functions, J. London Math. Soc., (2) 28 (1983), 57-61.

[6] F. Gross and C. C. Yang, The fix-points and factorization of meromorphic functions, Trans. Amer. Math. Soc., 168 (1972), 211-219.

[7] W. K. Hayman, Meromorphic functions, Oxford Mathematical Monographs (1964), Clarendon Press, Oxford.

[8] _ The local growth of power series: A survey of the Wiman-Valiron method, Canad. Math. Bull., (3) 17 (1974), 317-358. 
[9] P. C. Rosenbloom, The fix-points of Entire Functions, Medd. Lunds Univ. Mat. Sem. Suppl.-Bd. M. Riesz, (1952), 187-192.

[10] A. Schönhage, Über das Wachstum zusammengesetzter Funktionen, Math. Z., 73 (1960), 22-44.

[11] N. Steinmetz, Über die faktorisierbaren Lösungen gewöhnlicher Differentialgleichungen, Math. Z., 170 (1980), 169-180.

[12] G. Valiron, Lectures on the General Theory of Integral Functions, Edouard Privat, Toulouse, 1923.

[13] C. C. Yang, Further results on the fix-points of composite transcendental functions, J. Math. Anal. Appl., 90 (1982), 259-269.

[14] - On the fix-points of composite transcendental entire functions, J. Math. Anal. Appl., 108 (1985), 366-370.

Received May 31, 1988 and in revised form November 11, 1988. Research performed as a Feodor Lynen Research Fellow of the Alexander von Humboldt Foundation.

Cornell University

ITHACA, NY 14853

Current address: Technical University of Aachen

Lehrstuhl II für Mathematik

D-5100 Aachen, Federal Republic of Germany 


\section{PACIFIC JOURNAL OF MATHEMATICS EDITORS}

\author{
V. S. VARADARAJAN \\ (Managing Editor) \\ University of California \\ Los Angeles, CA 90024-1555-05 \\ Herbert Clemens \\ University of Utah \\ Salt Lake City, UT 84112 \\ Thomas ENRIGHT \\ University of California, San Diego \\ La Jolla, CA 92093
}

R. FINN

Stanford University

Stanford, CA 94305

Hermann FlaschKa

University of Arizona

Tucson, AZ 85721

VAughan F. R. Jones

University of California

Berkeley, CA 94720

Steven Kerckhoff

Stanford University

Stanford, CA 94305
RobION KIRBY

University of California

Berkeley, CA 94720

C. C. MOore

University of California

Berkeley, CA 94720

HAROLd STARK

University of California, San Diego La Jolla, CA 92093

\begin{tabular}{|c|c|c|c|c|}
\hline & ASSOCIA & E EDI & & \\
\hline R. ARENS & $\begin{array}{l}\text { E. F. BECKENBACH } \\
(1906-1982)\end{array}$ & NeumanN & $\begin{array}{c}\text { F. WoLF } \\
(1904-1989)\end{array}$ & K. Yoshida \\
\hline & SUPPORTINC & INSTI & TIONS & \\
\hline UNIVERSITY & ARIZONA & UNIVERS & OF OREGON & \\
\hline UNIVERSITY & BRITISH COLUMBIA & UNIVER & OF SOUTHEI & CALIFORNIA \\
\hline CALIFORNIA & STITUTE OF TECHNOLOGY & STANFOI & UNIVERSITY & \\
\hline UNIVERSITY & CALIFORNIA & UNIVER & OF HAWAII & \\
\hline MONTANA S & TE UNIVERSITY & UNIVER & OF TOKYO & \\
\hline UNIVERSITY & NEVADA, RENO & UNIVER & OF UTAH & \\
\hline NEW MEXIC & TATE UNIVERSITY & WA & N STATE UN & ERSITY \\
\hline OREGON ST & UNIVERSITY & & OF WASHINC & $\mathrm{ON}$ \\
\hline
\end{tabular}




\section{Pacific Journal of Mathematics}

\section{Vol. 143, No. $1 \quad$ March, 1990}

Walter Bergweiler, On the fix-points of composite functions $\ldots \ldots \ldots \ldots \ldots 1$ Aldo Biancofiore, Maria Lucia Fania and Antonio Lanteri, Polarized surfaces with hyperelliptic sections $\ldots \ldots \ldots \ldots \ldots \ldots \ldots \ldots \ldots$

Ciprian Borcea, Deforming varieties of $k$-planes of projective complete

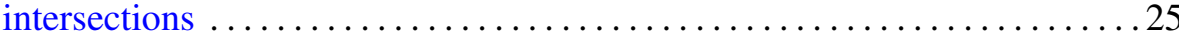

Morton Brown, Fixed points for orientation preserving homeomorphisms of

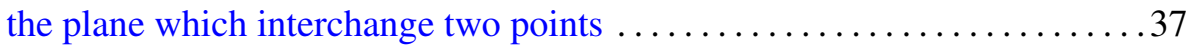

Hao Zhi Chuan, Note on the inequality of the arithmetic and geometric means

Paul Henry Edelman and Dennis E. White, Codes, transforms and the

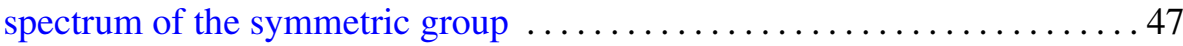

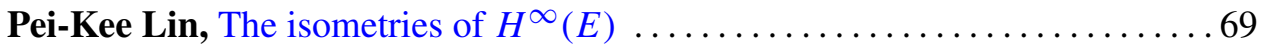

James J. Moloney, Residue class domains of the ring of convergent

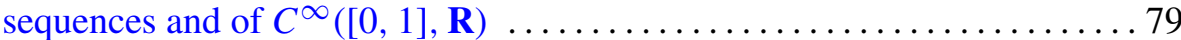

Zhong-Jin Ruan, The structure of pure completely bounded and completely

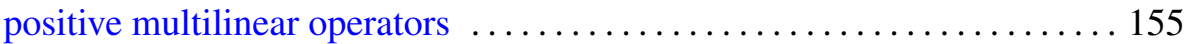

Wolfgang Ruess and William H. Summers, Weakly almost periodic semigroups of operators

Gideon Schwarz, A pretender to the title "canonical Moebius strip" . . . . . . 195

Ryszard Szwarc, Banach algebras associated with spherical representations of the free group 